CASE II.-The age of this patient was four years. During the preceding summer she had been affected with an obstinate porriginous eruption on the scalp; and, as this had disappeared, hooping cough set in and had affected her general health greatly, not only by the frequently repeated and severe paroxysms of coughing, but also by an almost constant irritability of the stomach, so that all food was rejected, as soon as swallowed.

A plaster of hemlock and Burgundy pitch, sprinkled with five grains of the antimonial tartrate, when applied between the shoulders, and a mixture composed of a mild bitter infusion, with the addition of syrup of poppies and of cinchona powder, was ordered to be given in repeated doses during the day. In 24 hours, the plaster was removed, for it had already induced a copious eruption of pustules. The sickness bad quite ceased ; and the cough was greatly mitigated. No second application was necessary ; and this girl, in the course of a week or two, was pronounced to be well.

Several other cases, all of which evince most satisfactorily the admirably curative effects of the plaster we have described, are detailed by $\mathrm{Dr}$. Corsin. He is inclined to explain the modus operandi of this external treatment, on the principle that there is in most cases of looping cough, a tendency to the occurrence of some cutaneous eruption, whether this tendency be of spontaneous development, or whether it is the result of the retrocession of a pre-existent exanthem. It has indeed been often remarked that the severity of pertussis is frequently much mitigated on the supervention of impetigo, or porrigo._Lancette Française.

\title{
PROTRACTED ADHESION OF A PORTION OF THE PLACENTA, WITH FINAL SLOUGHING AND SEPARATION.
}

BY JOHN A. SWETT, M.D. NEW YORK.

Prior to his departure for Europe, Dr. Swett placed in my hands"a Case Book containing a collection of observations made by him in his dispensary practice. From this I have selected the following case. It is a singular one in showing, in the first place, the length of time that may elapse between the birth of a child and the total separation of the placenta ; in the second, the symptoms that such a preternatural adhesion may occasion; and fally, the changes that the placenta may undergo, or at least a part of it, previous to its separation.

While the case was in progress, it wa's not, for some time, thoroughly understood. Several physicians examined it with the Doctor, and the general impression, I think, was, that the diseased appearance was the consequence of some change of structure in the cervix uteri itself. The feeling produced by the dry slough was peculiar, and might be compared to that from a piece of dried sponge.

New York, Sept. 29th, 1835.

John Watson, M.D.

Mrs. Duffie, a healthy looking Irish woman, aged 25, was visited on the 28th of May, 1834. About six months before this time, she had, 
after a labor of twelve hours, given birth to her first child. She was atlended, as she states, by an ignorant person, by whom the soft parts were very much injured, and she has been ailing ever since. Her symptoms when first seen, and for some time previous, were the following:

Pain and tenderness in the loins, extending along the sacrum and around the hips. This she calls an aching tired feeling. It is not so severe in the morning, especially before rising, as towards night, - but the difference is not considerable. Dysuria to a great extent; tenderness, pain, and a sensation of weight about the fundament ; tenderness across the abdomen; most perceptible in the epigastrium.

On examination, per vaginam, the parts about the fossa navicularis were found thickened and hard,-contracting the external orifice; the pelvis was capacious ; the uterus had prolapsed, with its neck inclining towards the sacrum, and the fundus resting against the bladder.

Within a few days, although still nursing her infant, the patient had menstruated. The discharge was darker than natural, and was followed by a leucorrhœa, which is still upon her, and is attended with an offensive smell. Her general health is somewhat reduced; she is pale and feeble; appetite impaired. She is inclined to nausea, with flatulence, and sense of oppression; bowels constipated; pulse small and feeble, no febrile excitement.

She was directed 01 . Ricini, 13 . as a laxative; to restrict herself to farinaceous food; and to use a saturnine lotion.

June 2d.-The leucorrhœal discharge had changed its color and smell, becoming sanious and putrid. The os tincæ no longer to be felt, in consequence of a dry spongy mass of no great sensibility occupying the cervix uteri. This mass, through the speculum vaginæ, appeared black, and attached to the womb. The vagina as far as noticed, was healthy. The patient had now some fever, with occasional attacks of faintness, but her strength and spirits were not much further reduced.

June 7 th. - The slough was found to be confined to the anterior margin of the os tincæ and cervix uteri; the nouth of the uterus apparently elongated but closed. In the course of the vagina were several small yellow elevated spots, some of which were ulcerated. The patient complained of a throbbing about the coccyx. She was directed to use a lotion of chloride of soda, with laxative medicine and farinaceous diet. For two days previous to this, she had used an injection of diluted nitric acid-using at the same time the acid internally.

July 23d.-General health improved-the slough not occupying more of the healthy surface than formerly, but larger, and more projecting. Saw the patient with Doctor F. U. Johnston-concluded to do nothing further than to keep the patient on simple regimen, and to watch the natural progress of the case.

August 18th.-Patient able to take exercise, and has ventured to Staten Island. For three weeks no discharge from vagina; but there is still occasional throbbing about the coccyx.

August 30th.- In making an examination, found the slough entirely separated. It was easily removed by the finger. It had probably lain detached in the vagina for some time, but prevented from being discharged by the contraction of the orifice. The slough was black, dry 
and tough. The lips of the os tincæ now appeared natural, and without tenderness-the uterus still prolapsed with the neck pressing towards the recturn. The patient of late had been over-working herself, and suffered from gastric irritation, with some febrile excitement. For the purpose - of regaining her health she went for a short time to the country. During the severe period of her illness, and up to the early part of July, she had menstruated regularly, but she had an abortion on the 7th of October.

\section{United States Medical and Surgical Journal.}

\section{SURGICAL CASES AT THE UNIVERSITY OF BRESLAU.}

Prof. Benedict, of the above University, has published in Rust's Magazine a review of the surgical clinique for the years 1828 to 1833 inclusive. The facts connected with the medical and surgical practice in large hospitals are always valuable, and we avail ourselves of every means within our reach of presenting such to our readers. We have room this week only for the reports on lithotomy and cancer.

Lithotony.- - This operation was performed during the six years, thirteen times; once on a girl of twelve years, the rest on males, the oldest of whom had reached the age of fifty-three. All these patients were cured, with the exception of four, none of whom died immediately after the operation. Thus one of these four, a boy sixteen years of age, had been dismissed cured from the establishment, but died eleven weeks after of typlious fever. The second died fourteen day's after the operation, when the left kidney was found in a state of suppuration, and the right one engorged. In the third case, death on the 11 th day, evidenced suppuration of the left kidney, extending down to the pelvis. The fourth case was fatal on the fourth day from peritonitis. In reference to lithotomy, Professor Benedict relates a very curious case, which, on account of its termination, is worthy of rerord. The patient, fifty-three years of age, who had long suffered from symptoms of stone, was received into the hospital in 1816, but left it without an operation having been performed. After a lapse of twelve years the patient presented hinself again, but during this time the calculus bad acquired such a magnitude, that whenever the sound was passed between it and the bladder, it became locked. It was thought searcely possible to remove the stone by an operation; however, this was undertaken, and the incision being prolonged considerably towards the rectum (which was not injured), the calculus was extracted after its outer shell had given way under the forceps. The stone weighed seven and a half ounces, without counting several fragments that were lost. On the fifth day the patient was seized with low typhous fever, without any signs of inflammation of the urinary or abdominal organs. The usual stimulants seemed of no avail, when the author accidentally learned that his patient was a confirmed brandy drinker. All other means were at once laid aside, and the patient given a tablespoonful of brandy every two hours. This treatment was followed by such happy results, that in four days the quantity of brandy could be diminished, and the patient was content with a glass at breakfast. The patient was discharged cured after some months. 